International Journal of Engineering \& Technology, $7(2.20)(2018) 159-163$
International Journal of Engineering \& Technology
SPC
Website: www.sciencepubco.com/index.php/IJET
Research paper

\title{
A hybrid elman neural network predictor for time series prediction
}

\author{
N. Mohana Sundaram ${ }^{1} *$, S. N. Sivanandam ${ }^{2}$ \\ ${ }^{1}$ Faculty of Computer Science and Engineering, Karpagam Academy of Higher Education, Coimbatore, India \\ ${ }^{2}$ Professor Emeritus, Karpagam college of Engineering, Coimbatore, India \\ *Corresponding author E-mail: itismemohan@gmail.com
}

\begin{abstract}
Artificial Neural Networks have become popular in the world of prediction and forecasting due to their nonlinear nonparametric adaptive-learning property. They become an important tool in data analysis and data mining applications. Elman neural network due to its recurrent nature and dynamic processing capabilities can perform the prediction process with a good range of accuracy. In this paper an Elman recurrent Neural Network is hybridised with a time delay called a tap delay line for time series prediction process to improve its performance. The Elman neural network with the time delay inputs is trained tested and validated using the solar sun spot time series data that contains the monthly mean sunspot numbers for a 240 year period having 2899 data values. The results confirm that the proposed Elman network hybridised with time delay inputs can predict the time series with more accurately and effectively than the existing methods.
\end{abstract}

Keywords: Elman Neural Networks; Time Series Prediction; Solar Sun Spot Numbers; Artificial Neural Networks.

\section{Introduction}

A time series data is a sequence of data values presented over a time period. Sun spot counts, ocean tide data, the stock price movement or index movement data and most of the physical, chemical and medical data are considered to be time series data. Time series prediction is the process of predicting future values on a parameter based on previously observed values of physical, chemical, financial or medical systems by constructing a dynamic predictive model. The prediction process becomes a complex problem because of the non linear and the dynamic behaviour of the parameters existing in a physical system.

In the time series problem the future values of a time series $p(t)$ are predicted based on the past values. This form of prediction is called nonlinear autoregressive, or NAR, and can be written as follows:

$$
\mathrm{p}(\mathrm{t})=\mathrm{f}(\mathrm{p}(\mathrm{t}-1), \ldots, \mathrm{p}(\mathrm{t}-\mathrm{d}))
$$

The Elman neural network is best suited for modelling the dynamic behaviour of non linear systems for prediction because of the recurrent link layer or the feed back layer which can store or retain the detailed past information. Static networks do not have any feedback loops. Dynamic neural networks may have feedback loops or time delay or both. Dynamic networks are preferable for time series as they have memory in the form of loops or delay lines. The hybrid Elman-time delay network proposed in this work has both feedback loop and time delay inputs.

The Elman Networks [2] have an additional layer known as Context layer into which the hidden layer neurons are copied and are fed back to hidden layer during the next cycle which was proposed by JEFFREY L.ELMAN [Elman 1990].

Fig 1 shows an Elman network.

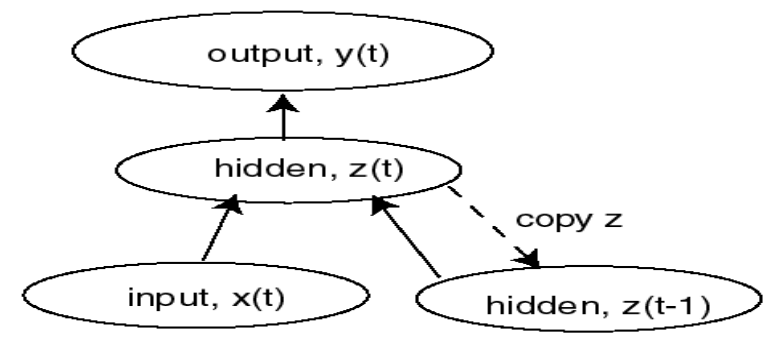

Fig. 1: The Elman Neural Network with Context Layer.

Time delay networks are feed-forward networks with the input weights having tap delay lines associated. Due to these tap delay lines they have a finite dynamic response to input time series data.

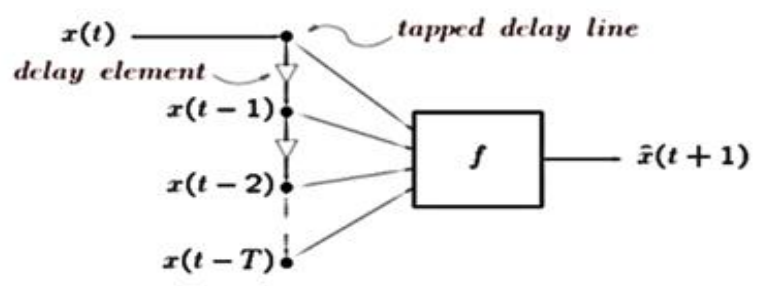

Fig. 2: Time Delay Network.

The past Information is inserted by increasing the input space in to a fixed size blocks. This is called a tapped delay line because inputs are arranged in a delayed manner and discretely shifted as time passes. They are primarily applied to work on sequential data Fig2 shows a Time delay Network.

In this paper the Elman network is incorporated with time delay inputs so that both the properties of dynamic networks namely the memory in the form of feed back loop and the time delay exist in 
the model. Fig 3. shows the Elman network hybridised with time delays.

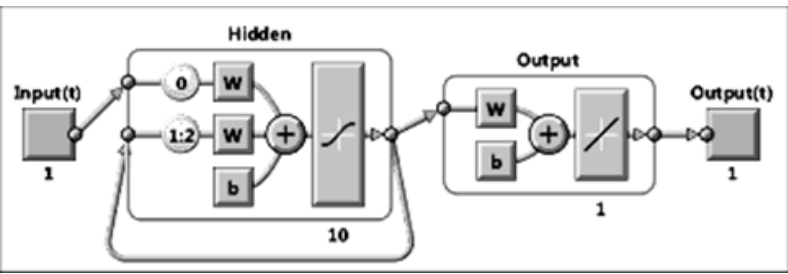

Fig. 3: Elman Neural Network Hybridised with Time Delay Inputs.

\section{Sunspot data}

The sunspots are basically visible surfaces within the Sun that are pock-marked with black flecks. Of all solar features, the sunspots are the most easily observed and their intermittent appearance has numerous impacts on Earth.

The solar sun spot data set contains the monthly mean sunspot numbers for a 240 year period from January of 1749 to July of 1990. It has 2899 values recording 2899 months of mean solar sunspots. The Fig 4 shows plot of the 2899 sun spot values the data (source: SILSO Data/Image, Royal Observatory of Belgium, Brussels)

Source: (SILSO data/image, Royal Observatory of Belgium, Brussels)

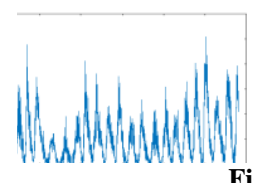

Fig. 4: The Solar Sun Spot Data.

\section{Related work}

Many Researchers employed artificial neural Networks for prediction for various applications.

M.Ardalani-Farsa and S.Zolgafhari (2010) performed the sunspot forecasting using Hybrid Elman-NARX using residual analysis and they had an MSE of 1.19 E-02 Ajabshirizadeh A et al (2011) [4] used neural networks to analyze the time series of solar activity. They predicted the solar index (Rz) in solar cycle 24 . As per their predictions of yearly sunspot number, the maximum of cycle 24 will occur in the year 2013 and will have an annual mean sunspot number of 65 . They used feed-forward neural network model with LM algorithm Zhihang Tang, Rongjun Li ( 2011) [5] proposed a new improved neural network called Hybrid feedback Elman network. Back propagation algorithm is used to train Elman network generally which makes the network convergence slowly. The proposed hybrid feed back method speed up the original ENN algorithm and offered a good performance for the prediction tasks.

Tamer Khatib et al (2012) [7] performed the prediction of hourly solar radiation values for Kuala Lumpur using generalized regression neural network (GRNN), the feed-forward back propagation neural network (FFNN), the cascade-forward back propagation neural network (CFNN), and the Elman back propagation neural network (ELMNN).Their results show that GRNN has a higher performance compared to the other proposed networks.
Moghaddamnia.A et al., (2009) [9] performed a comparative analysis on different prediction model namely local linear regression (LLR), multi layer perceptron (MLP), Elman neural network, neural network auto-regressive model with exogenous inputs (NNARX) and adaptive neuro-fuzzy inference system(ANFIS) using the solar radiation data. They have established that LLR and NARX models have better estimation capabilities.

Rohitash Chandra and MengjieZhang (2012) [8] proposed SLCCRNN (Synapse Level Cooperative co evolution of Elman recurrent neural network) and NL-CCRNN (Neural Level Cooperative co evolution of Elman recurrent neural network) for chaotic time series prediction. They used Mackey-Glass, Lorenz and Sun spot time series data and proved the SL-CRNN and NL- CRNN methods perform well compared to Multi-layer perceptron, Elman RNN , FIR network, Wavelet packet multilayer perceptron, Radial basis network with orthogonal least squares, Locally linear neurofuzzy model and Hybrid NARX-Elman RNN with residual analysis.

Chuanjin Jiang and Fugen Song (2011) employed [10] Nonlinear Autoregressive with Exogenous input (NARX) Neural networks, Back propagation Neural networks (BP ) and ARIMA models for prediction of sunspot number Chaotic Timeseries.

They considered the sunspot numbers of 310 years from 1700 to 2009 as chaotic time series sample, studied the internal chaos and calculated embedding dimension and delay. Later they designed the model with NARX neural network and conducts prediction. They established from the results that compared with BP Network and ARIMA Model, NARX network can better predicts the time series.

Junru GAO, Yuqing Wang (2013) employed Elman neural networks for fault diagnosis of steam turbine. They have analyzed the steam turbine data to predict the type of fault out of ten types of faults namely Rotor unbalance(F1), Aerodynamic coupling(F2), shaft misalignment(F3), oil whirl(F4), Rotor radial rub (F5),Symbiotic Loosening(F6), Thrust bearing damage(F7),surge (F8), Bearing pedestal looseness (F9) and Range bearing stiffness (F10). They conducted the simulations using Elman neural network and feed forward network and concluded that the Elman Network ehibits a better performance

Qiuwang Wang et al (2006) employed artificial neural networks for heat transfer rate Prediction of shell and tube heat exchangers with segmental baffles. They declare that the difference between the experimental data and predicted results was less than $2 \%$. They recommended that neural networks can be easily used to predict the performances of thermal systems in engineering applications, especially to model heat exchangers for heat transfer analysis.

Artificial Neural Network (ANN) approach [12] for reservoir water level prediction using daily data of inflow, water level and release was performed by Shilpi Rani and Dr. Falguni Parekh (2014). They developed three networks namely Cascade, Elman and Feed-forward back propagation and evaluated their performance using 23 years of hydrological data.

The three types of artificial neural networks, namely, Feed forward networks, Elman networks and Cascade forward networks were built for forecasting wind speeds and directions by Nicolus K Rotich et al (2014). All the models were trained with real data of wind speeds and directions collected over a period of two years. They declared that Elman networks returned the lowest errors.

Chandra .R (2015) presented a competitive Cooperative Coevolution method for training Elman neural networks for chaotic time-series prediction. They proposed two different instances of the competitive which employed different decomposition methods to enforce island-based competition. Their work established improved performance in the proposed methods in most cases when compared with standalone CC and other methods.

Nicolus K. Rotich et al (2014) [15] employed neural networks for wind source forecasting and assessment.

Jujie Wanga et al (2014) proposed novel empirical mode decomposition with Elman Neural Network (EMD-ENN) approach, a hybrid of empirical mode decomposition (EMD) and Elman neural 
network (ENN), is to forecast wind speed. They used four prediction cases of wind speed to evaluate the performance of the proposed hybrid approach. They compared the results with the with the back-propagation neural network, and Elman neural network .They established that the proposed EMD-ENN model has the minimum mean absolute error, mean square error, and mean absolute percentage error.

Zhihang Tang, Rongjun Li (2011) [18] proposed a new improved neural network called Hybrid feedback Elman network. Back propagation algorithm is used to train Elman network generally which makes the network convergence slowly. The proposed hybrid feed back method speed up the original ENN algorithm and offered a good performance for the prediction tasks.

Hossam Adel Zaqoot et al (2001) employed Multilayer perceptron (MLP) and radial basis function (RBF) neural networks for prediction of the $\mathrm{pH}$ value of Seawater along Gaza Beach and obtained MSE values 0.009 for MLP and 0.002 for RBF networks.

Mohana Sundaram.N et al (2016) employed Elman neural network to predict the mortality due to pollution.

Mohana Sundaram N and Renupriya (2016) V employed the Elman neural network for the prediction of $\mathrm{pH}$ value of the solution in the $\mathrm{pH}$ neutrlization process.

María C. Palancar et al (1998) designed control system with two artificial neural networks (ANN's) for the $\mathrm{pH}$-neutralization of acidic liquid streams.

Harwinder Kaur \& Dalwinder Singh Salaria (2013) conducted an analysis to evaluate performance of different training algorithms of neural network in software effort estimation. Their results proved that Bayesian Regularization algorithm offered better accuracy than other training algorithms.

\section{Implementation of solar sun spot data pre- diction using hybrid ENN}

The Elman network hybridised with time delay inputs is simulated in Matlab 12 software.

The architecture of Elman Predictor is shown in TABLE 1

Table 1: Architecture of the Elman Predictor

\begin{tabular}{l}
\hline No of Input Neurons \\
\hline No of Output Neurons \\
No of Hidden Layers \\
No of neurons in the \\
hidden layer \\
Training Algorithm \\
Activation Function
\end{tabular}

1
1
1

27

Bayesian Regularization Back Propagation Tan-Sigmoid for input-hidden layer Linear

\begin{tabular}{ll}
\hline & function for hidden-output layer \\
Performance & RMSE, Regression \\
\hline
\end{tabular}

There is no hard and fast rule for setting the number of hidden layer neurons. The experiment is repeated with different number of hidden layer neurons as 5, 7, 10, 15,27and 40 and the performance is studied. The best results are obtained with one hidden layer with 27 neurons.

\subsection{Performance metrics for data prediction}

The metrics used for the data prediction process are

- MSE ( Mean Squared Error) / RMSE ( Root Mean Squared Error).

- Regression.

The error is measured as:

Error $=\mathrm{e}=($ actual value - predicted value $)$

The Mean squared error is

$$
F=m s e=\frac{1}{N} \sum_{i=1}^{N}\left(e_{i}\right)^{2}=\frac{1}{N} \sum_{i=1}^{N}\left(t_{i}-\alpha_{i}\right)^{2}
$$

$\mathrm{N}$ - The total number of data points.

\subsection{Results and discussion}

The observed values of the proposed Hybrid Elman Neural Network are MSE $=0.0002997$ and Regression $\mathrm{R}=0.9967$.

The TABLE 2 shows the comparison of the results available in literature with the proposed method.

Table 2: Comparison of the MSE Values of Solar Sun Spot Data

\begin{tabular}{llll}
\hline $\begin{array}{l}\text { S. } \\
\text { no }\end{array}$ & Method & MSE & Literature \\
\hline 1 & NARX network & 0.0004 & \\
2 & Feed forward BP & 0.0081 & Chuanjin Jiang and Fugen \\
3 & network & Song (2011) \\
4 & ARIMA method & 0.0061 & \\
\hline
\end{tabular}

The comparison chart for the MSE of the sun spot data for different approaches are shown in figure 5

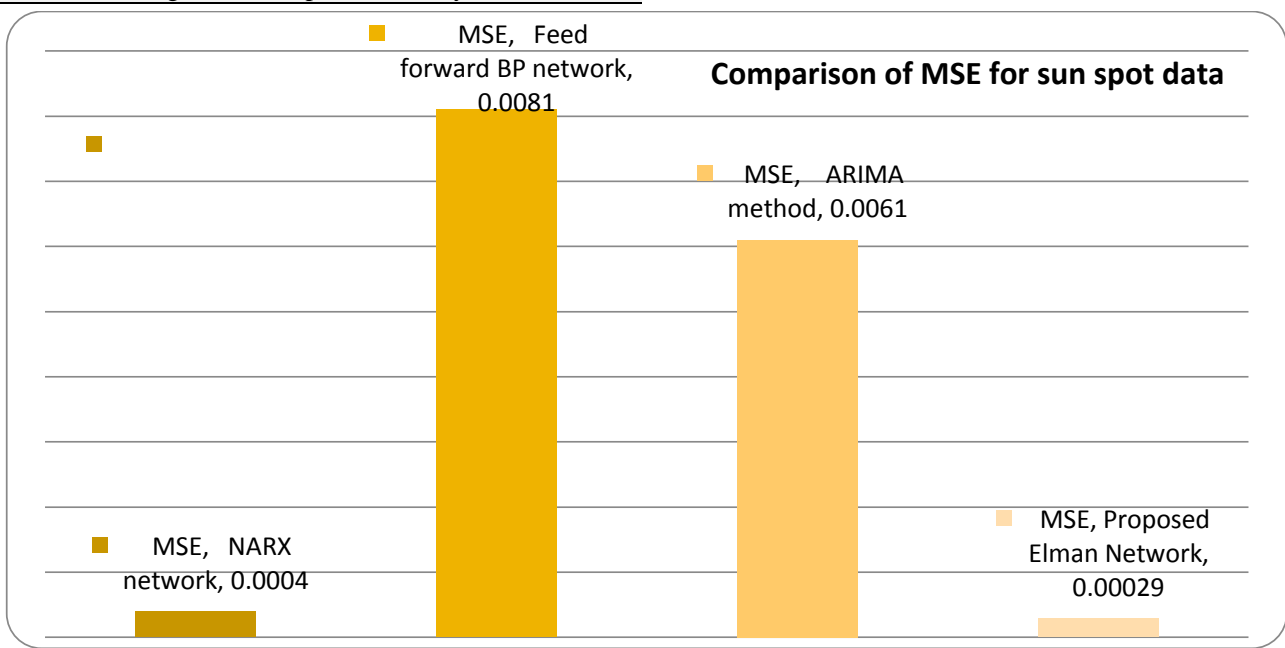

Fig. 5: Comparison Chart for Solar Sun Spot Data.

Table 3: Comparison of Performance of Solar Sun Spot Data

\begin{tabular}{llll}
\hline S. no & Method & RMSE & \\
\hline 1 & CCRNN-Synapse Level & 1.66 E-02 & Rohitash Chandra and Mengjie Zhang(2012) \\
2 & CCRNN-Neuron Level & 2.66 E-02 & Ardalani-Farsa, M., \& Zolfaghari, S. (2010). \\
3 & Hybrid Elman-NARX Network with residual analysis & 1.19 E-02 & Ard \\
\hline
\end{tabular}




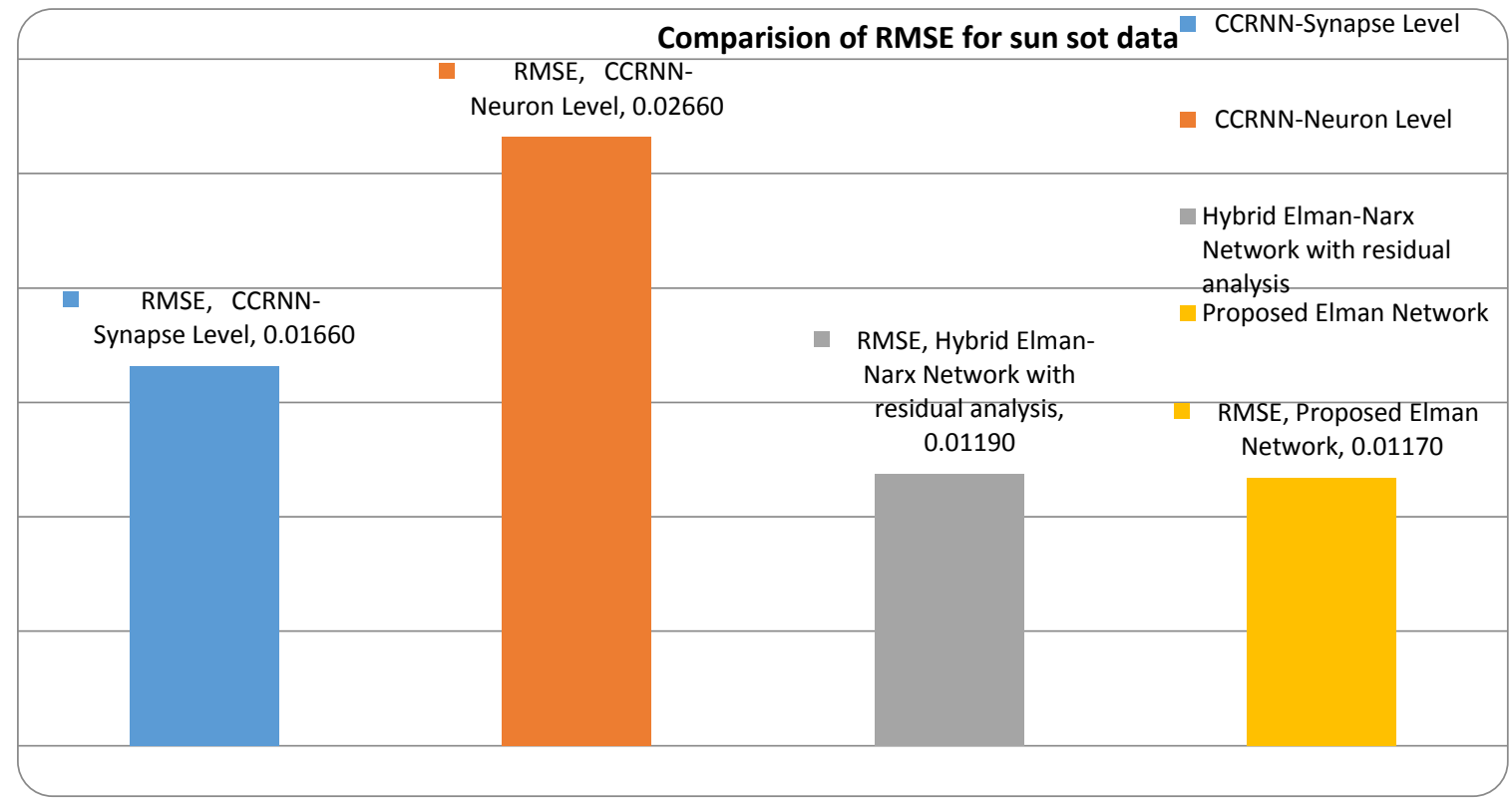

Fig. 6: Comparison of RMSE of Sun Spot Data.

The RMSE values are compared with the values obtained in this work and are tabulated in TABLE 3.

The figure 6 shows the comparison of RMSE of sun spot data. Referring to the Tables 2 and 3 and the figures 5 and 6 it is evident that the proposed hybrid Elman network has a lowest MSE of 0.00029 and an RMSE of 0.0117 , which are lower, compared to the other method shown in literature. Further, the regression value is 0.9967 which shows that the values predicted by the Hybrid Elman network follows the actual values.

\section{Conclusion}

The Hybrid Elman neural network hybridised with the time delay inputs was developed and its performance is studied by applying the benchmark sun spot numbers data. Because of its dynamic adaptive learning capabilities the proposed Hybrid Elman network exhibited better performance. The artificial neural networks especially the hybridised Elman- Time delay network is most suited for time series data prediction which includes many applications in engineering, medical and financial fields.

\section{References}

[1] Ardalani-Farsa, M., \& Zolfaghari, S.Chaotic time series prediction with residual analysis method using hybrid Elman-NARX neural networks. Neurocomputing, 73(13), 2540-2553,201

[2] Ajabshirizadeh A, N. Masoumzadeh Jouzdani and Shahram Abbassi , Neural network prediction of solar cycle 24, Research in Astronomy and Astrophysics. 2011 Vol. 11 No. 4, 491-496

[3] Bharath Chandra Mummadisetty, Astha Puri, Ershad Sharifahmadian, Shahram Latifi, A Hybrid Method for Compression of Solar Radiation Data Using Neural Networks, International Journal of Communications, Network and System Sciences, 2015, 8, 217-228.

[4] Brunelli U., V. Piazza, L. Pignato, F. Sorbello, S. Vitabile, Three hours ahead prevision of $\mathrm{SO} 2$ pollutant concentration using an Elman neural based forecaster, Building and Environment 43 (2008) 304-314.

[5] Chandra .R , Competition and Collaboration in Cooperative Coevolution of Elman Recurrent Neural Networks for Time-Series Prediction, IEEE Transactions on Neural Networks and Learning Systems, vol. 26, no. 12, pp. 3123-3136, Dec. 2015.

[6] Chuanjin Jiang and Fugen Song, Sunspot Forecasting by Using Chaotic Timeseries Analysis and NARX Network, Journal of Computers, VOL. 6, 2011, 1424-1429

[7] ELMAN, J. L. Finding Structure in Time. Cognitive Science 14, 2 (1990), 179-211.
[8] Harwinder Kaur \& Dalwinder Singh Salaria, Bayesian Regularization Based Neural Network Tool for Software Effort Estimation, Global Journal of Computer Science and Technology Neural \& Artificial Intelligence Volume 13 Issue 2, 2013,44-50.

[9] Hossam Adel Zaqoot, Ahsanullah Baloch, Abdul Khalique Ansari, and Mukhtiar Ali Unar, Application of Artificial Neural Networks for Predicting Ph in Seawater Along Gaza Beach, Applied Artificial Intelligence, 24:667-679,2001, Taylor \& Francis Group, LLC ISSN: 0883-9514 print :1087-6545.

[10] Jujie Wanga, Wenyu Zhangb, Yaning Lic, Jianzhou Wangc, Zhangli Dangb , Forecasting wind speed using empirical mode decomposition and Elman neural network, Applied Soft Computing, Volume 23, October 2014, Pages 452-459

[11] Junru GAO, Yuqing Wang, The Research on the Methods of Diagnosing the Steam Turbine Based on the Elman Neural Network, Journal of Software Engineering and Applications, 2013, 6, 87-90.

[12] María C. Palancar, José M. Aragón, José S. Torrecilla, pH-Control System Based on Artificial Neural Networks, Industrial \& Engineering Chemistry Research 199837 (7), 2729-2740

[13] Moghaddamnia.A , R.Remesan , M.HassanpourKashani , M.Mohammadi , D.Han , J.Piri , Comparison of LLR, MLP, Elman, NNARX and ANFIS Models - with a case study in solar radiation estimation, Journal of Atmospheric and Solar-Terrestrial Physics, 71 (2009) 975-982.

[14] Mohana Sundaram.N ,S. N. Sivanandam and R.Subha, Elman Neural Network Mortality Predictor for Prediction of Mortality Due to Pollution, International Journal of Applied Engineering Research ISSN 0973-4562 Volume 11, Number 3 (2016) pp 1835-1840.

[15] Mohana Sundaram N, Renupriya V ,Nonlinear Predictive Model of Chemical Process System Using an ElmanNeural Network, International Journal of Engineering Science and Computing, DOI 10.4010/2016.504, ISSN 2321 3361, Volume 2016, Issue February, pp 2069-2073.

[16] Nicolus K. Rotich, Jari Backman, Lassi Linnanen and Perfilieve Daniil, Wind Resource Assessment and Forecast Planning with Neural Networks, Journal of Sustainable Development of Energy, Water and Environment Systems, 2014, Volume 2, Issue 2, pp 174 190

[17] Qiuwang Wang, Gongnan Xie, Ming Zeng, Laiqin Luo, Prediction of heat transfer rates for shell and tube heat exchangers by artificial neural networks approach, Journal of Thermal Science 2006, 15(3):257-262.

[18] Rohitash Chandra and MengjieZhang, Cooperative coevolution of Elman recurrent neural networks for chaotic time series prediction, Neurocomputing 86(2012)116-123.

[19] Shilpi Rani and Dr. Falguni Parekh, Predicting Reservoir Water Level Using Artificial Neural Network, International Journal of Innovative Research in Science, Engineering and Technology, Vol. 3 Issue 7, July 2014, ISSN: 2319-8753.

[20] Tamer Khatib, AzahMohamed, K.Sopian and M.Mahmoud, Assessment of Artificial Neural Networks for Hourly Solar Radiation 
Prediction, International Journal of Photoenergy, Volume 2012, Article ID 946890, pages 1-7.

[21] Zhihang Tang, Rongjun Li, an Improved Neural Network Model and Its Applications, Journal of Information \& Computational Science 8: 10 (2011), 1881-1888. 